\title{
COMUNIDADES DE ROEDORES DE PAMPAS DE ALTURA EN LAS SIERRAS GRANDES EN CÓRDOBA, ARGENTINA
}

\section{RODENT COMMUNITIES OF HIGH PAMPAS IN THE SIERRAS GRANDES OF CORDOBA, ARGENTINA.}

\author{
M. B. Kufner ${ }^{1}$, G. Gavier y D. Tamburini
}

\section{Resumen}

Se estudió la composición de comunidades de roedores, su abundancia relativa y su relación con la estructura del hábitat, en las pampas de altura de las Sierras Grandes de Córdoba. Se establecieron cuatro sitios de muestreo, dos en la Pampa de San Luis a 1900 msnm, con pendientes suaves, céspedes resultantes de pastoreo y matas y herbáceas estivales. Los otros dos en un área escarpada y rocosa, con pajonales y pastizales bajos cerca de Cuchilla Nevada, entre 1700 y $1800 \mathrm{msnm}$. Estacionalmente, cuadrículas de trampas Sherman se activaron durante tres noches consecutivas. La cobertura de la vegetación se estimó como descriptor del hábitat. Tres especies fueron registradas: Akodon spegazzinii Thomas, 1897, Phyllotys xanthopygus (Waterhouse, 1837) y Oligoryzomys flavescens (Waterhouse, 1837). La abundancia relativa de la comunidad es mayor en invierno y otoño y en la primera zona que reúne características más "amigables". A. spegazzini hace uso generalista del hábitat, mientras que $P$. xantophygus es más abundante en el sitio escarpado rocoso. El sobrepastoreo afecta el hábitat y la comunidad. Estos datos y los de otros autores sobre las comunidades de roedores del macizo de las Sierras Grandes, permiten pensar en una estructura característica por encima de los $1700 \mathrm{~m}$ de altitud.

Palabras clave: Comunidades roedores, Pampas de altura, Hábitat, Sierras Grandes, Córdoba.

\begin{abstract}
The composition of rodent communities, and their relative abundance and relationship with habitat structure, were studied in the high pampas of the Sierras Grandes, Córdoba, Argentina. Four sampling places were set: two in Pampa de San Luis at $1900 \mathrm{~m}$ above sea level, a place with soft slopes covered by short and tall grasses and summer herbs. The two other sites were sharp and rocky, with tall and low grassland, near Cuchilla Nevada between 1700 and $1800 \mathrm{~m}$ above sea level. Grids of 20 Sherman traps distanced $10 \mathrm{~m}$ each, were activated during three consecutive nights, seasonally during 1997 and 2000. Vegetation cover was assessed as habitat descriptor. With a capture effort of 876 night traps, three species were registered: Akodon spegazzinii Thomas, 1897, Phyllotys xanthopygus (Waterhouse, 1837) y Oligoryzomys flavescens (Waterhouse, 1837). The relative abundance in the community was higher in winter and autumn, and in the first area that gathers more "friendly" characteristics. A. spegazzini uses habitat in a generalist way, while $P$. xantophygus prefers the rocky place. Overgrazing affects both, habitat and community. Our data and other authors' about rodents of Sierras Grandes Mountains, suggest a characteristic community structure above $1700 \mathrm{~m}$ altitude.
\end{abstract}

Key words: Rodent communities, High Pampas, Habitat, Sierras Grandes, Cordoba.

\section{Introducción}

Las sierras de Córdoba caracterizadas por su variedad topográfica y fisonómica sustentan una diversidad de especies que Polop (1989) ha mencionado de importancia zoogeográfica y evolutiva. La estructura del hábitat influye en la organización y distribución de las comunidades de mamíferos; variaciones altitudinales de la diversidad de roedores han sido comprobadas en los diferentes pisos de vegetación de las sierras (Polop, 1989; Kufner et al., 1998; Altrichter et al., en prensa). En el macizo de las Sierras Grandes se han encontrado semejanzas entre comunidades de roedores de la Pampa de Achala -parte central mencionada frecuentemente como "isla biogeográfica"- y de la zona más austral del mismo, la sierra de Comechingones (Polop, 1991; Priotto et al., 1996).

$\mathrm{Se}$ analizan las comunidades de roedores y su relación con el hábitat en los pastizales de altura de la Pampa de San Luis, ubicada en la porción Norte de dicho macizo. Ello colaborará asimismo a dilucidar algunos patrones corológicos pseudoandinos. La intervención humana en el hábitat complejiza, sin embargo, la interpretación de los procesos y relaciones referidos. En este sentido, se investiga la influencia sobre la diversidad, del pastoreo como actividad dominante en el área. 


\section{Materiales y métodos}

Área de estudio. Según Capitanelli (1979) en las Sierras Grandes el clima es semihúmedo, con invierno térmico, sin verano y escaso déficit hídrico, prevaleciendo atributos típicos de altura: temperaturas bajas, heladas, vientos fuertes, nevadas en invierno y lluvias concentradas en verano. El promedio anual de precipitaciones es de $840 \mathrm{~mm}$, con oscilaciones marcadas. La temperatura media anual es de $14.5^{\circ} \mathrm{C}$. La amplitud térmica diaria puede ser importante, descendiendo la temperatura entre $15^{\circ}$ y $20^{\circ} \mathrm{C}$ en pocas horas.

La vegetación se distribuye en pisos altitudinales. Aproximadamente desde los $1000 \mathrm{msnm}$ aparece el piso de "pastizales serranos" que se desarrolla sobre distintos tipos de roca. Los pastizales se componen de "céspedes" de juncáceas, ciperáceas, especies rastreras y arrosetadas; los "pajonales" de gramíneas altas en penacho y los "pastizales bajos" con menor porte y mayor diversidad (Luti et al., 1979; Cabido et al., 1989).

El área de estudio está ubicada en la Pampa de San Luis en las Sierras Grandes de la provincia de Córdoba, en el Dpto. Cruz del Eje. Los sitios de muestreo se ubicaron en dos lugares separados por una distancia aproximada de $10 \mathrm{~km}$. La Zona I se encuentra al SW de la Pampa de San Luis, a 1900 msm; consta de altiplanicies o valles inclinados levemente hacia el E, de escasa pendiente, relieve ondulado y sectores de suelo profundo y superficial. La vegetación consiste en céspedes resultantes del pastoreo y matas y herbáceas estivales. La Zona II está cercana a la localidad de Cuchilla Nevada, entre 1 700 y $1800 \mathrm{msnm}$. Se trata de un hábitat escarpado y heterogéneo, con pendientes marcadas y afloramientos de rocas metamórficas. La vegetación consta de pajonales y pastizales bajos.

Metodología. La composición de comunidades de roedores y su abundancia relativa, así como su relación con la estructura del hábitat se determinó en dos áreas representativas de la vegetación característica de las pampas de altura. En cuatro sitios de muestreo apareados, se establecieron otras tantas cuadrículas de 20 trampas tipo Sherman en un arreglo de 4x5 trampas, distanciadas entre sí 10 metros. Las cuatro grillas utilizadas permitieron abarcar diversos microhábitats como roquedales, distintas condiciones de pastizales y diferentes pendientes o relieve. En cada sitio -en 1997 y en 2001- se activaron estacionalmente las trampas, durante tres noches consecutivas. La composición de la comunidad se determinó en base al registro de todas las especies capturadas. La identificación taxonómica fue realizada teniendo en cuenta caracteres externos y análisis electroforéticos.

El índice de abundancia relativa se estimó corrigiendo el número de individuos según el esfuerzo de captura realizado en cada sitio; se dividió por el número de noches trampas y se multiplicó por doscientos, obteniéndose un valor estandarizado que permitió establecer comparaciones. Las diferencias en abundancia relativa por especies, por sitios, por estaciones y para el muestreo total se evaluaron mediante un test $\chi 2$. El uso del hábitat se estimó a partir de la relación entre la abundancia relativa de micromamíferos y la cobertura vegetal (M'Closkey \& Fieldwick, 1975).

En cada grilla se evaluó la cobertura de la vegetación según el método de Canfield (Hays et al., 1981). En tres transectas de 30 metros de longitud se midió en todos los sitios la cobertura comprendida entre el suelo y un metro de altura, considerando los siguientes estratos de vegetación: E1 $=0$ a $0.05 \mathrm{~m}, \mathrm{E} 2$ $=0.06$ a $0.20 \mathrm{~m}, \mathrm{E} 3=0.21$ a $0.50 \mathrm{~m}$ y E4= más de $0.50 \mathrm{~m}$. Asimismo se estimó el porcentaje de roca y suelo expuesto. Estas mediciones llevadas a cabo en invierno y primavera de 1997 y 2000, permitieron analizar las diferencias en la estructura de la vegetación entre las Zonas I y II y el cambio entre los dos períodos estudiados.

\section{Resultados \\ Estructura del hábitat}

La vegetación de la Zona I presentó pastizales no muy altos (Tabla 1a) y proporciones de roca expuesta entre un 70 y $80 \%$ más bajas que la Zona II. Ésta con una fisonomía más escarpada que la anterior se caracterizó por pasturas más densas y altas (Tabla 1b) alternando con roquedales.

Tabla 1. Cobertura de la vegetación (\%) por estratos.

a) Zona $\mathrm{I}$.

\begin{tabular}{|c|c|c|c|c|}
\hline Estrato & $\begin{array}{l}\text { Invierno } \\
1997\end{array}$ & $\begin{array}{l}\text { Invierno } \\
2000\end{array}$ & $\begin{array}{l}\text { Primavera } \\
1997\end{array}$ & $\begin{array}{l}\text { Primavera } \\
2000\end{array}$ \\
\hline $\begin{array}{llll}d e & 0 & a & 5 \\
\mathrm{~cm} & & & \end{array}$ & 41.09 & 37.66 & 47.18 & 39.58 \\
\hline $\begin{array}{l}\text { de } 5 \text { a } 20 \\
\text { cm }\end{array}$ & 10.78 & 31.7 & 9.11 & 22.48 \\
\hline $\begin{array}{l}\text { de } 20 \text { a } 50 \\
\mathrm{~cm}\end{array}$ & 14.29 & 30.64 & 21.41 & 37.94 \\
\hline $\begin{array}{l}>\text { de } 50 \\
\mathrm{~cm}\end{array}$ & 33.84 & 0.00 & 22.29 & 0.000 \\
\hline
\end{tabular}

b) Zona II.

\begin{tabular}{|c|c|c|c|c|}
\hline Estrato & $\begin{array}{l}\text { Invierno } \\
1997\end{array}$ & $\begin{array}{l}\text { Invierno } \\
2000\end{array}$ & $\begin{array}{l}\text { Primavera } \\
1997\end{array}$ & $\begin{array}{l}\text { Primavera } \\
2000\end{array}$ \\
\hline $\begin{array}{llll}\text { De } & 0 & a & 5 \\
\mathrm{~cm} & & & \end{array}$ & 24.40 & 33.76 & 28.18 & 35.04 \\
\hline $\begin{array}{l}d e \quad 5 \text { a } 20 \\
\mathrm{~cm}\end{array}$ & 6.38 & 6.96 & 6.49 & 11.27 \\
\hline $\begin{array}{l}\text { de } 20 \text { a } 50 \\
\mathrm{~cm}\end{array}$ & 56.43 & 41.85 & 65.33 & 40.12 \\
\hline $\begin{array}{l}>\text { de } 50 \\
\mathrm{~cm}\end{array}$ & 12.79 & 17.44 & 0.00 & 13.57 \\
\hline
\end{tabular}


Las dos zonas de estudio difirieron tanto en fisonomía como en la estructura de la vegetación. La cobertura se comparó por estaciones, en forma apareada, en cada zona (Invierno 1997, $\chi 2=68.31 ; 5$ g.1.; $\mathrm{P}<0.001 ;$ Invierno 2000, $\chi 2=50.91 ; 5$ g.l.; $\mathrm{P}<0.001$; Primavera 1997, $\chi 2=55.36 ; 5$ g.1.; $\mathrm{P}<0.001$; Primavera 2000, $\chi 2=29.50 ; 5$ g.l.; $\mathrm{P}<0.001)$.

sitio 1 (Zona I)

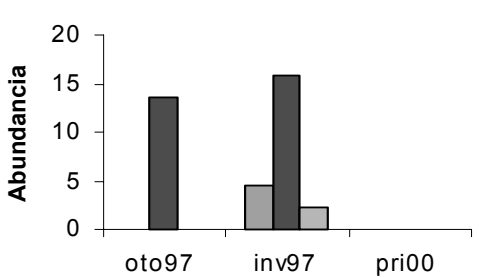

sitio 3 (Zona II)

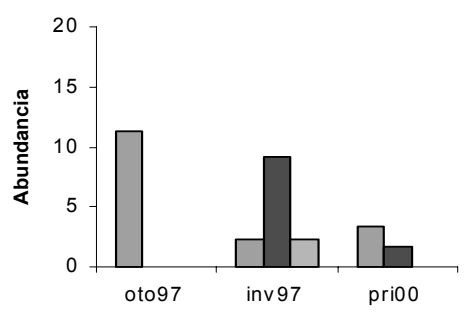

de la región. Ocurrió lo mismo en la Zona II $(\chi 2=$ 10.35; 3 g.l.; $\mathrm{P}<0.02$ ).

En el año 2000 no se comprobaron diferencias estacionales significativas en la vegetación de ninguna zona, probablemente en relación con uniformización de la vegetación provocada por un intenso pastoreo. Ello se puso de manifiesto en las diferencias registradas en la vegetación de cada zona, al comparar

sitio 2 (Zona I)

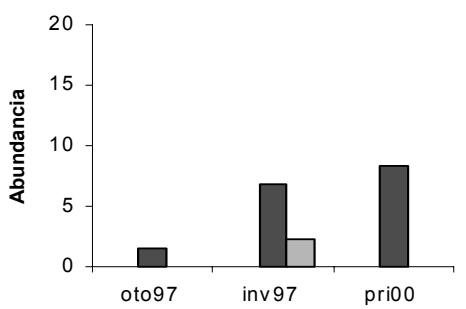

sitio 4 (Zona II)

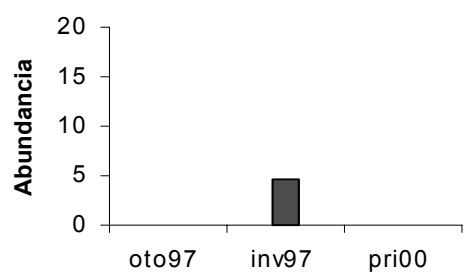

$\square$ P. xantophygus

$\square$ A. alterus

$\square$ O. flavescens

Figura 1. Evolución de la abundancia específica en los hábitats de las Zonas I y II.

Tabla 2. Comparación de la estructura estacional de la vegetación de ambas zonas, en los dos períodos estudiados.

\begin{tabular}{|c|c|c|c|c|}
\hline \multirow{2}{*}{ Período } & \multicolumn{2}{|l|}{ Zona I } & \multicolumn{2}{|l|}{ Zona II } \\
\hline & $\begin{array}{l}\text { Invierno } \\
2000\end{array}$ & $\begin{array}{l}\text { Primavera } \\
2000\end{array}$ & $\begin{array}{l}\text { Invierno } \\
2000\end{array}$ & $\begin{array}{l}\text { Primavera } \\
2000\end{array}$ \\
\hline $\begin{array}{l}\text { Invierno } \\
1997\end{array}$ & $\begin{array}{l}\chi^{2}=47.49 \\
3 \text { g.l. } \\
P<0.001\end{array}$ & & $\begin{array}{l}\chi^{2}=13.20 \\
3 \text { g.1. } \\
P<0.001\end{array}$ & \\
\hline $\begin{array}{l}\text { Primavera } \\
1997\end{array}$ & & $\begin{array}{l}\chi^{2}=30.73 \\
3 \text { g.l. } \\
P<0.001\end{array}$ & & $\begin{array}{l}\chi^{2}=15.83 \\
3 \text { g.l. } \\
P<0.001\end{array}$ \\
\hline
\end{tabular}

La estructura de la vegetación mostró variación temporal en ambas zonas de estudio, cuando se comparó los dos períodos estudiados. En 1997 la vegetación de invierno de la Zona I difirió significativamente de la del verano $(\chi 2=31.42 ; 3$ g.l.; $\mathrm{P}<0.02$ ), coincidiendo con la fenología característica los períodos 1997 y 2000 (Tabla 2). Evidencia notable de la degradación de estos pastizales de altura la constituyen las cárcavas de erosión halladas, algunas con más de $4 \mathrm{~m}$ de profundidad y $5 \mathrm{~m}$ de ancho y alrededor de $200 \mathrm{~m}$ de extensión.

Comunidades de roedores

Con un esfuerzo de captura de 876 noches trampas, un total de 47 roedores fueron colectados. Los mismos se distribuyeron en tres especies: Akodon spegazzini $(\mathrm{n}=34)$, Phyllotis xantophygus $(\mathrm{n}=10) y$ Oligoryzomys flavescens $(\mathrm{n}=3)$. La abundancia relativa fue mayor en invierno y otoño $\left(\chi^{2}=10.74,2\right.$ g.l., $\mathrm{P}<0.0001)$ y en el sitio 1 de la Zona I, seguido del sitio 3 de la Zona II $\left(\chi^{2}=13.05,3\right.$ g.l., $\mathrm{P}<$ $0.0001)$. A. spegazzini fue la especie más capturada comparada con $P$. xantophygus $\left(\chi^{2}\right.$ para contingencia de proporciones $2 \times 2=0.777, \mathrm{P}<0.001$ ) (Figura 1). La distribución de la abundancia de especies en los distintos sitios indica que $A$. spegazzini hace un uso generalista del hábitat, mientras que $P$. xantophygus, si bien aparece en dos sitios, fue capturado principalmente en el sitio escarpado con roca expuesta. $O$. flavescens fue capturado en los sitios 1, 2 y 3 con sólo un individuo en cada uno. La proporción 
de individuos capturados fue significativamente mayor en 1997 con respecto al 2000 ( $\chi^{2}$ para diferencia de proporciones $2 \times 2=0.57, \mathrm{P}<0.001$ ).

\section{Discusión}

Los resultados sugieren que, comparativamente, la Zona I reúne características de hábitat más "amigables" para la comunidad de micromamíferos estudiada; éstas serían su homogeneidad, continuidad espacial y provisión de recursos, al menos durante 1997. Al cabo de tres años solamente, se advierte el efecto de la degradación del hábitat principalmente por sobrepastoreo (pérdida de suelo y de cobertura vegetal, modificación de la vegetación, entre otros), sobre la comunidad de roedores. Ello se evidencia en cambios en la representatividad y riqueza específicas en los distintos hábitats.

Según Polop (1989) A. spegazzini se distribuiría en bosques de tabaquillos (Maytenus boaria) en quebradas y acantilados. Nuestros datos apoyan una mayor plasticidad de esta especie, que hace un uso generalista del hábitat. Coincidentemente con el mismo autor, $P$. xantophygus es encontrado en zonas rocosas. El resto de las especies citadas para las Sierras Grandes son en general raras y aportan a la diversidad: Reithrodon sp., Oxymycterus sp. y Oligoryzomys flavescens (Polop, 1989; Priotto et al., 1996). La última especie es también constatada en bajo número en este trabajo.

Polop (1989) señala que la Pampa de Achala sería una isla con elementos principalmente andino patagónicos y guayano brasileños, presentado en este sentido una discontinuidad con el resto de la provincia de Córdoba. Priotto et al. (1996), por su parte, discuten esta exclusividad en base al hallazgo de comunidades semejantes en Sierra de Comechingones, al Sur. Nuestros resultados completan el relevamiento de múridos del macizo de las Sierras Grandes en su porción septentrional y aportan información sobre las características de la mastocenosis. Éstas permiten confirmar la existencia de comunidades de roedores con estructura particular por encima de los $1700 \mathrm{~m}$ de altitud. La comprobación de esta situación en otros puntos de las Sierras Pampeanas sería de interés.

\section{Agradecimientos}

A N. Gardenal, J. Polop y M. Torres por identificar las especies. A J. Baldo y Y. Arzamendia por colaborar en el trabajo de campo. A. R. Altamirano por facilitarnos lugar en el campo que administra. A
O. Pierantonelli por su apoyo en los viajes.

\section{Literatura citada}

Altrichter M., Kufner M., Giraudo L., Gavier G., Tamburini D. \& Sironi M. 2001. Comunidades de micromamíferos de bosque y pastizal de la Reserva La Quebrada, Sierras Chicas de Córdoba. Abundancia, diversidad y relación con la estructura del hábitat. Vida Silvestre Neotropical. En prensa.

Cabido M., Acosta A. \& Díaz S. 1989. Estudios fitosociológicos en los Pastizales de las Sierras de Córdoba, Argentina. Las comunidades de la Pampa de San Luis. Phytocoenologia. 17(4): 569592.

Capitanelli R.G. 1979. Clima. En: Geografía Física de la Provincia de Córdoba. Vázquez, J.B.; R. Miatello y M.E. Roqué (Directores). Boldt. Pp. 464. Bs. As.

Hays R., Summers C. \& William S. 1981. Estimating wildlife habitat variables. Fish \& Wildlife Service. FWS/OBS-81/47.

Kufner M.B., Giraudo L., Gavier G., Tamburini D., Altrichter M. \& Sironi M. 1998. Fauna de tetrápodos y sus biótopos en la Reserva La Quebrada, Río Ceballos, Córdoba. Acta Zoológica Lilloana. 44(1): 177-184.

Luti R., Solis M., Galera F., de Ferreyra N.M., Berzal M., Nores M., Herrera M. \& Barrera J. 1979. Vegetación. Páginas 297-367 en J. Vázquez, R. Miatello y E. Roqué, eds. Geografía Física de la Provincia de Córdoba. Editorial Boldt, Buenos Aires.

M'Closkey R. \& Fieldwick B. 1975. Ecological separation of sympatric rodents Peromyscus and Microtus. Journal of Mammalogy. 56: 119-129.

Polop J. 1989. Distribution and ecological observations of wild rodents in Pampa de Achala, Córdoba, Argentina. Studies on Neotropial Fauna and Environment. 24: 53-59.

1991. Distribución de Cricétidos en las sierras de Achala (Provincia de Córdoba, República Argentina). Rev. UNCR. 11: 115-121.

Priotto J.W., Morando M. \& Avila L. 1996. Nuevas citas de roedores de los pastizales de altura de la sierra de Comechingones, Córdoba, Argentina. Facena. 12: 135-137.

\footnotetext{
${ }^{1}$ CERNAR, F.C.E.F. y N. Vélez Sarsfield 299, 5000 Córdoba. bkufner@gtwing.efn.uncor.edu
} 